\title{
LAS JARRITAS DE SANTA MARIÑA DE AUGAS SANTAS (ALLARIZ), UNA APROXIMACIÓN A SU MORFOLOGÍA \\ THE JARS OF SANTA MARIÑA DE AUGAS SANTAS (ALLARIZ), AN APPROACH TOWARDS THEIR MORPHOLOGY
}

\author{
Francisco Alonso Toucido \\ Departamento de Historia \\ Facultad de Geografía e Historia \\ Universidad de Santiago de Compostela (USC) \\ 15782 Santiago de Compostela, A Coruña \\ franalonsotoucido@hotmail.es \\ M. Pilar Prieto Martínez \\ Departamento de Historia \\ Facultad de Geografía e Historia \\ Universidad de Santiago de Compostela (USC) \\ 15782 Santiago de Compostela, A Coruña \\ pilar.prieto@usc.es
}

\author{
Anxo Rodríguez Paz \\ Ayudante de Investigación \\ INCIPIT-CSIC \\ 15705 Santiago de Compostela, A Coruña \\ anxo.rodriguez@incipit.csic.es
}

Recibido: 01/11/2017

Aceptado: 06/04/2018

RESUMEN: Las jarras de Santa Mariña de Augas Santas son un conjunto de referencia excepcional debido a su número, tipología y contexto. Más de un ciento de jarritas medievales fueron localizadas a mediados del siglo XX en óptimo estado de conservación en el caño de desagüe de una sauna castreña, que hoy en día conforma la cripta de una basílica medieval inconclusa. La zona guarda una gran riqueza patrimonial tanto en materiales arqueológicos como a nivel de tradición oral. Las jarritas sobrepasan la centena contándose con varios tipos morfológicos y un gran número de las mismas se encuentran decoradas. Se presentan los resultados morfológicos del estudio realizado sobre las mismas y se comparan los mismos con otros contextos arqueológicos galaicos.

PALABRAS CLAVE: Plena Edad Media, Depósito ritual medieval, Orense, Tipología 
cerámica, procesos de producción

ABSTRACT: The jars of Santa Mariña de Augas Santas form an exceptional reference group due to their number, type, and context. A total of 128 mediaeval jars were found in the middle of the twentieth century in an excellent state of preservation in the drainage spout of a hill fort sauna, which today forms part of the crypt of an unfinished mediaeval basilica. The area contains a wealth of heritage both in terms of archaeological remains and oral traditions. The 122 jars that are still preserved belong to several morphological types, and a large number of them are decorated. We present the morphological results of the study carried out on them, and they are compared with other Galician archaeological contexts.

KEYWORDS: Middle Ages, mediaeval ritual deposit, Ourense, ceramic typology, production processes.

\section{INTRODUCCIÓN}

El entorno de Santa Mariña de Augas Santas constituye un referente excepcional para el estudio del pasado, gracias a su riqueza patrimonial arqueológica e inmaterial que propició intervenciones de puesta en valor desde mediados del siglo XX como las realizadas por el Conde-Valvís o Chamoso Lamas ${ }^{1}$, que publica su trabajo centrado en la cripta. Será en una de estas intervenciones cuando el caño de desagüe del Forno da Santa sea limpiado y se recuperen 128 jarras muy bien conservadas. A partir de su descubrimiento las jarras serán depositadas en la casa rectoral de la iglesia parroquial de Augas Santas y ocuparán alguna breve referencia en obras más amplias como 'España Visigoda' donde serán tipificadas como romanas². En los años 80 del siglo XX la Xunta de Galicia financia un estudio del cual se publicará un resumen ${ }^{3}$, finalmente una de las jarras se convierte en pieza del mes del Museo Arqueológico Provincial de Ourense $^{4}$ y la última publicación en la que se menciona será un trabajo de síntesis de Augas Santas 5 , posteriormente estas jarras quedarán en el olvido.

La investigación detrás de las jarras está reflejando la situación del estudio de la cerámica medieval para la región, hace 27 años. Suárez et al. ${ }^{6}$ realizan una síntesis de

1 CHAMOSO LAMAS, M. (1955). “Santa Marina de Augas Santas”. Cuadernos de Estudios Gallegos, X-20: 41-88.

2 MONTEAGUDO GARCÍA, L. (1967). “España Visigoda”. Noticiario Turístico, Suplemento 208: 3-63.

3 PEREIRA MARIÓN, C. (1991). “As cerámicas medievais de Santa María de Augas Santas (Allariz, Ourense)” Arqueoloxía, Informes 2, Campaña 1988, Xunta de Galicia: 347-350.

4 FARIÑA BUSTO, F. (2000): Xarriña Cerámica. Peza do Mes. Museo de Ourense, Setembro.

5 FARIÑA BUSTO, F. (2002): Santa Mariña de Augas Santas, Grupo Marcelo Macías. Ourense.

6 SUÁREZ OTERO, J., GIMENO GARCÍA-LOMAS, R. y FARIÑA BUSTO, F. (1989): “La cerámica medieval en Galicia”. En Avelino, J. y Bohigas, R. (eds.), La cerámica medieval en el norte y noroeste de la Península Ibérica. 
la cerámica medieval gallega para la que califican su estudio de "débil configuración", donde para momentos posteriores al siglo XI, ya se identificaba la jarra trilobulada. Hoy en día, pese a ciertos avances gracias a publicaciones llegadas, principalmente, de la arqueología profesional, el conocimiento sobre cerámica medieval gallega no ha cambiado en demasía ${ }^{7}$. Las publicaciones son reducidas principalmente para la cerámica altomedieval y plenomedieval siendo las bajomedievales las que han preocupado más en los últimos años ${ }^{8}$.

La jarra ha sido definida como uno de los fósiles directores de la Edad Media Plena Europea, debido a su constante presencia en yacimientos de esta cronología. Aunque la jarra más común es la de tipo trilobulado, cuyo origen se ha retrotraído hasta las jarras monoansadas de pico trilobulado abierto, de cronología romana ${ }^{9}$, las jarritas de Augas Santas, como veremos más adelante, presentan una mayor variabilidad.

En el caso gallego, se ha documentado la presencia de jarras en diversos yacimientos de época plenomedieval como por ejemplo en intervenciones realizadas en Santiago de Compostela. Durante la excavación en área del lazareto medieval de San Lázaro, se documentaron en sendos pozos jarras rotas in situ, de 20 y 30 centímetros de altura, presentando una de ellas una perforación en su cuello, el borde de estas jarras presenta una marcada tendencia al exvasamiento ${ }^{10}$. En otros puntos de la urbe medieval, como en la rúa do Franco n $^{\circ} 31$, también se identificaron tanto jarras como jarritas, estas últimas con evidencias de haber sido empleadas para cocinar ${ }^{11}$. En el yacimiento de Pousada, ayuntamiento de Santiago de Compostela, en el nivel de ocupación de los siglos VII-VIII se documenta una jarra ${ }^{12}$. Las jarritas de Santiago poco tienen que ver con las de Augas Santas, siendo las primeras muy achatadas y globulares, únicamente

Aproximación a su estudio. Secretariado de Publicaciones, Universidad de León, León: 285-301.

7 MARTínEZ PEÑíN, R. (2013). "Los estudios de cerámica medieval en el noroeste de la Península: Galicia y Norte de Portugal”. Interconexôes. Revista de Ciencias Sociais, 1(1): 33-60.

8 CÉSAR VILA, M., BONILLA RODRÍGEZ, A. y LÓPEZ PÉREZ, Ma . C. (2010): “Aportaciones al conocimiento de la cerámica producida en la última fase de la Edad Media en Galicia”. En Crespo, M. y Martínez, R. (eds.), Metodología de Análisis Aplicada los Estudios de Cerámica Tardoantigua y Medieval de la Península Ibérica, Lobo Sapiens, León: 145-160.

9 CÉSAR VILA, M., et al. (2010): “Aportaciones al conocimiento de la cerámica...”, op. cit., pág. 148.

${ }^{10}$ BONILLA RODRÍGUEZ, A. y CÉSAR VILA, M. (2005): "Excavación arqueológica en área en el solar de la antigua capilla y lazareto medieval de S. Lázaro (Santiago de Compostela, A Coruña)”. Gallaecia, 24: 219-242, pág. 228.

${ }^{11}$ ALONSO TOUCIDO, F.; PRIETO MARTÍNEZ M. P.; RODRÍGUEZ RESINO A. (2013). "Cerámica en silos. Contextos medievales e modernos na rúa do Franco no 31. Santiago de Compostela”. Gallaecia, 32: 215-248.

${ }^{12}$ BLANCO ROTEA, R., PRIETO MARTÍNEZ, M. P., BALLESTEROS ARIAS, P. y LÓPEZ GONZÁLEZ, L. F. (2010). "El despoblado de A Pousada: la formación de una aldea rural en la Alta Edad Media”. En Prieto Martínez, P. y Criado Boado, F. (Coord.), Reconstruyendo la historia de la comarca del Ulla-Deza (Galicia, España). Escenarios arqueológicos del pasado. TAPA 41. Santiago de Compostela: 111-120. 
podrían asemejarse a las de tipo olla; que describiremos más adelante. Las jarras son altas, de unos 30 centímetros o más, con decoración principalmente en base a un cordón digitado que aparte del rol decorativo, juega el de refuerzo de la pieza ${ }^{13}$.

Uno de sus rasgos característicos son las asas punzadas con varias incisiones verticales, tanto en las jarras como en las jarritas. En el Banco de España y otras intervenciones de la ciudad de Santiago, se han documentado este tipo de jarras y jarritas, tanto en época plenomedieval como bajomedieval, como es el caso del castillo de Rocha Forte, donde algunas jarras presentaban cocción oxidante ${ }^{14}$. En otros puntos de Galicia, también se han documentado jarras y jarritas, como es el caso de la Torre de Hércules, en A Coruña ${ }^{15}$, la iglesia medieval de As Encrobas en Cerceda ${ }^{16}$, o Roza das Aveas en Outeiro de Rei un túmulo prehistórico con presencia de material medieval en sus inmediaciones y una intervención de interés ya que se trata de una de las primeras en las que se realizan análisis arqueométricos a recipientes medievales en Galicia ${ }^{17}$. Los yacimientos más próximos a Augas Santas, con jarras medievales, se localizan en la ciudad de Ourense y San Vitor de Barxacova, una necrópolis asociada a una antigua capilla. En San Vitor de Barxacova, las jarras recuperadas presentan los punzados en el asa referidos anteriormente ${ }^{18}$. Con respecto a las jarras de la ciudad de Ourense son las que más se asemejan a las jarritas de Augas Santas, las jarritas son de reducido tamaño, forma troncocónica y tanto jarritas como jarras presentan incisiones cortas al lado de sus asas ${ }^{19}$, destacando una jarra bitroncocónica y jarra sin decorar tipo olla de As Burgas (ss. XIII-XV) ${ }^{20}$, una jarra tipo olla (ss. XII-XIV) y jarras trilobuladas decoradas (ss. XII-XIII) encontradas en el pozo de O Pompeo ${ }^{21}$; una jarra trilobulada

${ }^{13}$ ALONSO TOUCIDO, F. et al. (2013). “Cerámica en silos...”, op. cit., pág. 232.

${ }^{14}$ MARTÍNEZ CASAL, R. (2006). “A cerámica medieval da fortaleza da A Rocha Forte contribución ao seu estudo”, Gallaecia, 25: 187-225, pág. 208.

${ }^{15}$ BELLO DIÉGUEZ, J.Mª, SANJURJO SÁNCHEZ, J. y FERNÁNDEZ OSQUERA, D. (2008): “Los niveles medievales de la Torre de Hércules: caracterización arqueológica y datación mediante TL y OSL". Férvedes, 5: 453-464.

${ }^{16}$ CESAR VILA, M. y BONILLA RODRÍGUEZ, A. (2011): “Síntesis de los materiales cerámicos procedentes del yacimiento de As Encrobas (Cerceda - A Coruña)”. En González, S. (ed.), La cerámica en Galicia: de los castros a Sargadelos. Actas del XIV congreso anual asociación de ceramología. Asociación de ceramología: 143-152.

17 PRIETO MARTÍNEZ, M. P.; LANTES SUÁREZ, O.; VÁZQUEZ-LIZ, P.; MARTÍNEZ-CORTIZAS, A. (2010). "La cerámica de dos túmulos de Roza das Aveas (Outeiro de Rei, Lugo): Un estudio diacrónico del estilo y la composición”. BSAA, LXXVI: 27-62

${ }^{18}$ NIETO MUÑIZ, E.B. (2014): “Achádegos cerámicos na necrópole rupestres de San Vitor de Barxacova (Parada de Sil, Ourense)”. En De Man, A. e Tente, C (coords.) Estudos de cerâmica medieval. O norte e centro de Portugal, séculos IX a XII. Instituto de Estudos Medievais: 239-245.

${ }^{19}$ MAPO (Museo Arqueolóxico de Ourense) (2008): Ourense. A Cidade, da orixe ao século XVI. Catálogo de Exposición, Xunta de Galicia. Gráficas Garabal. A Coruña.

20 Ibid. pág. 41.

21 Ibid. pág. 57-58. 
decorada (ss. XI-XIII), una hucha decorada (ss. XIV-XV) en el antiguo Pazo do Bispo, y 4 jarritas del s. XIII registradas en la excavación de Rúa Colón 18-20-Rúa Cervantes 29 , idénticas a las encontradas en Augas Santas ${ }^{22}$. Como se puede ver la cronología de estas jarras es amplia desde el s. XI hasta el XIV y están basadas en estudios tipológicos y estratigráficos, pues no hay asociadas a ellas dataciones absolutas.

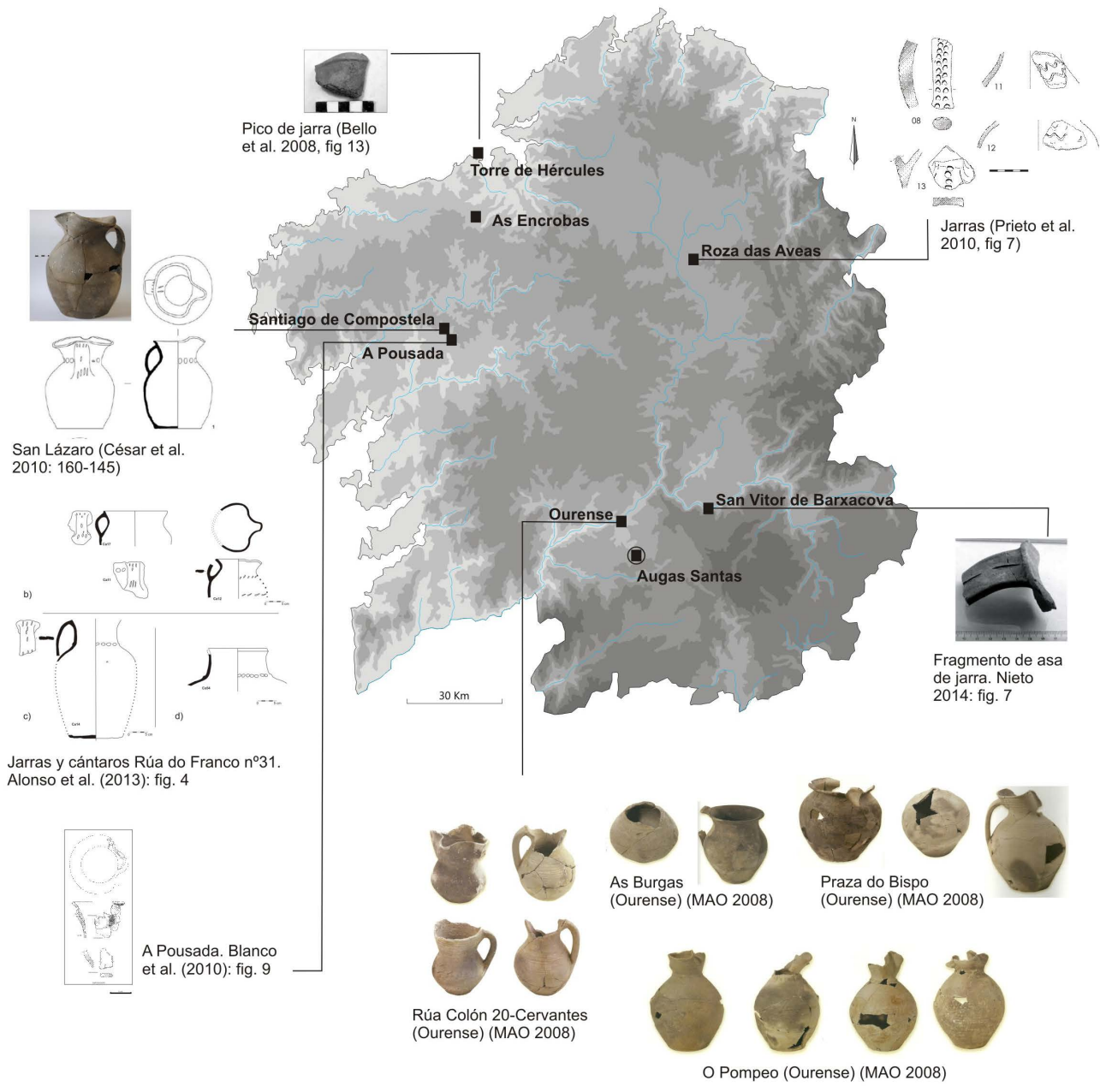

Fig. 1. Mapa de localización de Augas Santas y otros yacimientos de Galicia mencionados en el texto con jarras publicadas.

\footnotetext{
${ }^{22}$ Ibid. pág. 73; RODRÍGUEZ-CAO, C. (2009). "Escavación arqueolóxica en área nos soares n. 18-20 da rúa Colón e n 29 da rúa Cervantes da cidade de Ourense”. En F. Fariña Busto y M. Xusto Rodríguez (coord.): Ourense, A Cidade, da orixe ao século XVI. Catálogo de Exposición. Museo Arqueolóxico Provincial de Ourense, pp. 117-132.
} 


\section{MARCO GEOGRÁFICO Y ARQUEOLÓGICO}

Las jarras de Augas Santas se localizaron en la cripta de la Basílica da Ascensión y Os Fornos, situada en el lugar de Armea, parroquia de Santa Mariña de Augas Santas, ayuntamiento de Allariz, provincia de Ourense.

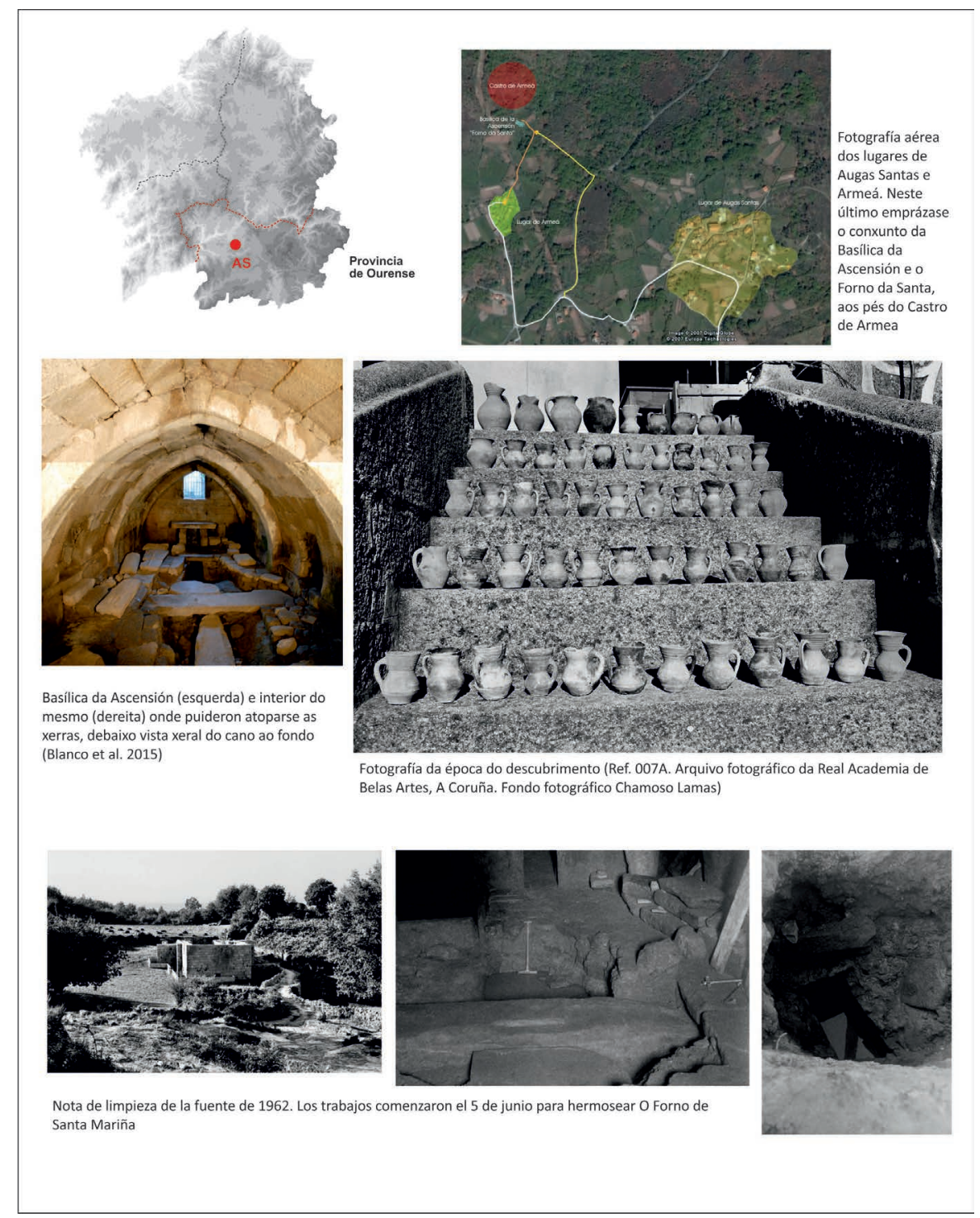

Fig. 2. Contexto de las jarras de Augas Santas. 
La iglesia se emplaza en una pequeña vaguada, sobre una terraza de origen antrópico, próxima al Castro o "Cibdá de Armea” y está delimitada al oeste por otra terraza, también de origen antrópico. Gracias a los estudios realizados recientemente (fig. 2) ${ }^{23}$, se ha constatado que el edificio fue construido al menos en 5 fases desde la Edad del Hierro hasta época contemporánea. En una fase inicial, en la Edad del Hierro, a las puertas del castro, se edifica un monumento son horno de tipo sauna. Posteriormente ese monumento es reformado en época tardoantigua y medieval. Destaca la fase en la que se construye una cripta, cronológicamente ubicada en el siglo XIII-XIV. En esta fase, se mantendría el uso del agua ya que, por una parte, se conservan las canalizaciones y el depósito dentro de la cripta y, por otra, se vincularía a ésta el depósito de jarras de cerámica datadas en el siglo XIII-XIV del presente trabajo ${ }^{24}$. En la parroquia se mantiene el patrimonio inmaterial vinculado al culto a santa Mariña, perviviendo en el paisaje numerosos topónimos y tradiciones relacionados con la santa y el culto al agua.

\section{RESULTADOS}

El depósito de Augas Santas originalmente estaba conformado por 128 jarras, de las cuales 6 están perdidas. El presente estudio, por lo tanto se realiza a partir de 122 jarras que presentan unas características estandarizadas que se pueden definir a través de 4 tipos morfológicos. Estos morfotipos han sido definidos a partir del rasgos más destacado de su perfil: Las jarras de cuello troncocónico (J1), las jarras tipo vaso (J2), las jarras tipo olla (J3) y las jarras con bocas lobuladas trilobular o cuatrilobular (J4). Desde el punto de vista de su conservación nos hallamos ante un conjunto excepcionalmente bien preservado, el mejor conservado de la región y probablemente de la Península Ibérica, pues 10 jarras (8\%) tienen el perfil completo y 97 (75\%) conservan el $90 \%$ del recipiente, excepcionalmente hay jarras que oscilan entre el $89 \%$ y el $50 \%$ de conservación del perfil, no existiendo jarras en peores condiciones de conservación. 22 jarras no conservan el asa (fig. 3).

\footnotetext{
${ }^{23}$ BLANCO ROTEA, R.; GARCÍA RODRÍGUEZ, S.; MATO-FRESÁN, C.; SANJURJO-SÁNCHEZ, J. (2015). "La Basílica da Ascensión y Os Fornos (Allariz, Ourense) y la cristianización de la arquitectura en la Antigüedad Tardía”. Estudos de Cuaternario, 12: 111-132.

24 PEREIRA MARIÓN, C. (1991). “As cerámicas medievais de Santa...”, op. cit.
} 


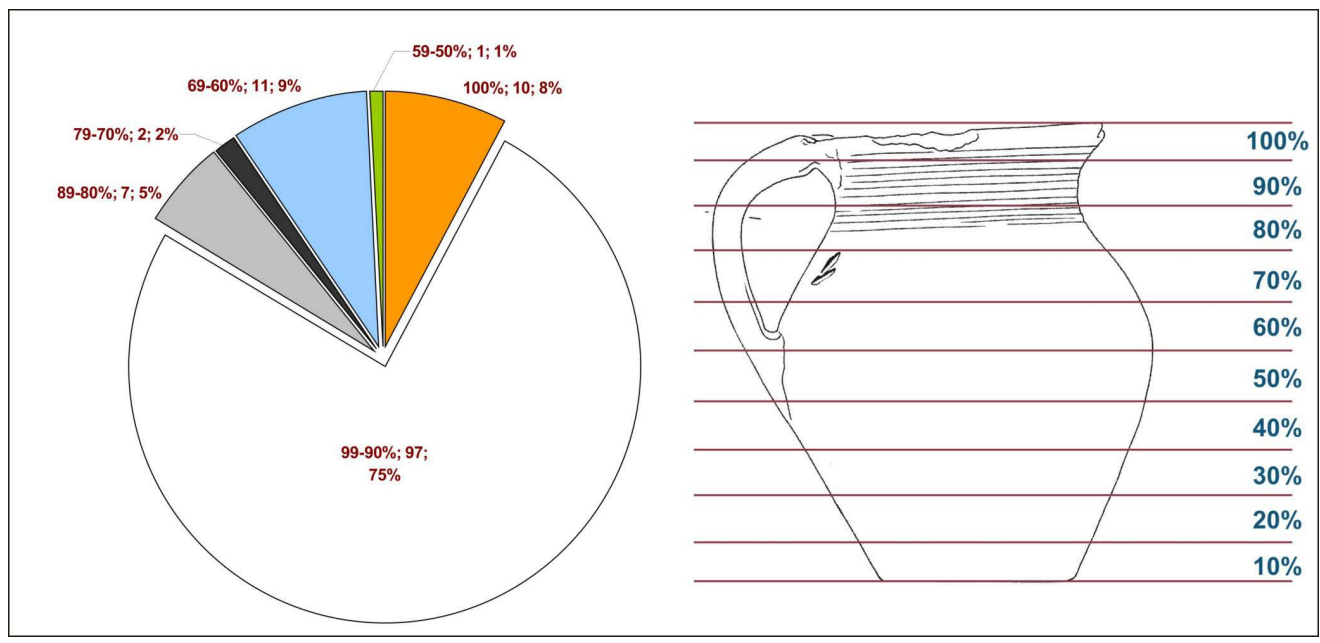

Fig. 3. Grado de conservación de las jarras y patrón utilizado para describir en porcentaje el tamaño del recipiente conservado.

\section{Tratamiento morfológico y tipos}

En lo que se refiere a los morfotipos podemos sintetizar las principales características morfológicas teniendo en cuenta tamaños, proporciones y asas. Todas las piezas están hechas a torno rápido y presentan un fondo plano y en todos los morfotipos predomina la simetría tanto en perfil como en altura. Las diferencias principales se apoyan en el grado de estrangulamiento de los cuellos y la forma y variedad de panzas en cada morfotipo (figs. 4 y 5 ).

J1 Jarras de cuello troncocónico (84 jarras - 70\%): los perfiles compuestos cerrados $^{25}$ (37 jarras), son más abundantes que los compuestos abiertos (27 jarras) y es en el único morfotipo en el que se observan perfiles compuestos rectos ( 3 jarras). Las bocas presentan una planta circular regular (62 jarras), es excepcional la existencia de irregularidad (3 jarras) aunque hay 19 jarras en las que no se sabe con seguridad el grado de regularidad de su boca. Los cuellos son esbeltos de paredes divergentes muy estranguladas. En su parte inferior se constatan 5 variantes de panzas: con carena (2), carena central (12), hombro central aplanado (4), hombro central (26) y globular (39). Los tamaños oscilan entre los recipientes con $63 \mathrm{~mm}$ de diámetro de boca y 85 mm de altura en el recipiente de menor tamaño y 86 mm de diámetro de boca y 124

${ }^{25}$ Los perfiles cerrados tienen un diámetro de boca inferior a la máxima expansión de la panza. Los perfiles abiertos tienen un diámetro de boca superior y los perfiles rectos poseen un diámetro de boca igual a la máxima expansión de la panza. 
mm de altura en el de mayor tamaño. La capacidad en litros ${ }^{26}$ de estas jarras oscila entre los 100 y los $230 \mathrm{~cm}^{3}$. Es el morfotipo que posee una mayor proporción en la configuración del perfil y variedad de tamaños. Las asas parten de la zona central del cuello y se apoyan en la parte central de la panza, haciendo un perfil abastonado.

J2 Jarras tipo vaso (20 jarras - 16\%): los perfiles compuestos cerrados son predominantes (10 jarras) frente a los abiertos (1 jarra). Las bocas presentan una planta circular regular, es excepcional la existencia de irregularidad (4 jarras). Los cuellos presentan paredes paralelas esbeltas y poco estranguladas y las panzas presentan la mayor variedad de perfiles con 6 variantes: carena (1), carena central (4), globular (6), con hombro superior (2), ultrahemiesférica (2) con hombro central (7). Los tamaños oscilan entre los recipientes con $62 \mathrm{~mm}$ de diámetro de boca y $81 \mathrm{~mm}$ de altura en el recipiente de menor tamaño y $64 \mathrm{~mm}$ de diámetro de boca y $100 \mathrm{~mm}$ de altura en el de mayor tamaño. La capacidad en litros de estas jarras oscila entre los 125 y los $220 \mathrm{~cm}^{3}$. Es el segundo morfotipo que posee un perfil mejor proporcionado, como el J1, aunque con una menor variedad de tamaños que aquel. Las asas parten de la zona superior del recipiente, desde el borde, apoyándose en la zona central de la panza, haciendo un perfil con una curva suave semicircular.

J3 Jarras tipo olla (14 jarras - 11\%): únicamente se documentan perfiles compuestos cerrados. Las bocas presentan una planta circular regular y sólo en dos casos es trilobulada con el borde vuelto hacia el interior. Los cuellos presentan paredes paralelas cortas y ligeramente estranguladas y las panzas tienen 4 variantes en su perfil: carena central (1), globular (10), hombro superior (2), hombro central (1). Los tamaños oscilan entre los recipientes con $85 \mathrm{~mm}$ de diámetro de boca y $80 \mathrm{~mm}$ de altura en el recipiente de menor tamaño y $118 \mathrm{~mm}$ de diámetro de boca y $154 \mathrm{~mm}$ de altura en el de mayor tamaño. La capacidad en litros de estas jarras oscila entre los 200 y los $920 \mathrm{~cm}^{3}$, en general son las de mayor capacidad de los cuatro morfotipos, pues la mitad de los recipientes superan los $300 \mathrm{~cm}^{3}$. Es el único morfotipo que posee un perfil achaparrado, con una boca de tendencia más ancha en relación con su altura de tamaños. Las asas parten de la boca del recipiente resaltando un hombro superior y apoyándose en la parte superior de la panza.

J4 Jarras con bocas trilobular o cuatrilobular (4 jarras - 3\%): predominan los perfiles compuestos cerrados (3 jarras) sobre los abiertos (1 jarra). Son los únicos recipientes que presentan una boca con pitorro lobulado, bien trilobulado (en dos casos de los tres con botón superior), o bien cuatrilobulado en un caso. Los cuellos presentan paredes paralelas cortas y muy estranguladas y las panzas tienen 3 variantes en su

${ }^{26}$ Datos tomados de las piezas que están casi completas, es decir, que conservan un perfil entre un $90 \%-100 \%$. 
perfil: carena central (1), globular (2), hombro superior (1). Los tamaños oscilan entre los recipientes con $62 \mathrm{~mm}$ de diámetro de boca y $85 \mathrm{~mm}$ de altura en el recipiente de menor tamaño y $84 \mathrm{~mm}$ de diámetro de boca y $203 \mathrm{~mm}$ de altura en el de mayor tamaño. El perfil de dos jarras se corresponde con el tipo J1 y J2, siendo las de menor tamaño, mientras que las otras dos jarras tienen un perfil más típico de cántaro sin llevar a adquirir su tamaño, siendo jarras más esbeltas. La capacidad en litros de estas jarras oscila entre los 140 y los $950 \mathrm{~cm}^{3}$, alcanzando la mayor capacidad y tamaño una de las jarras de este grupo Este es el tipo que se documenta en las excavaciones de la ciudad de Ourense.

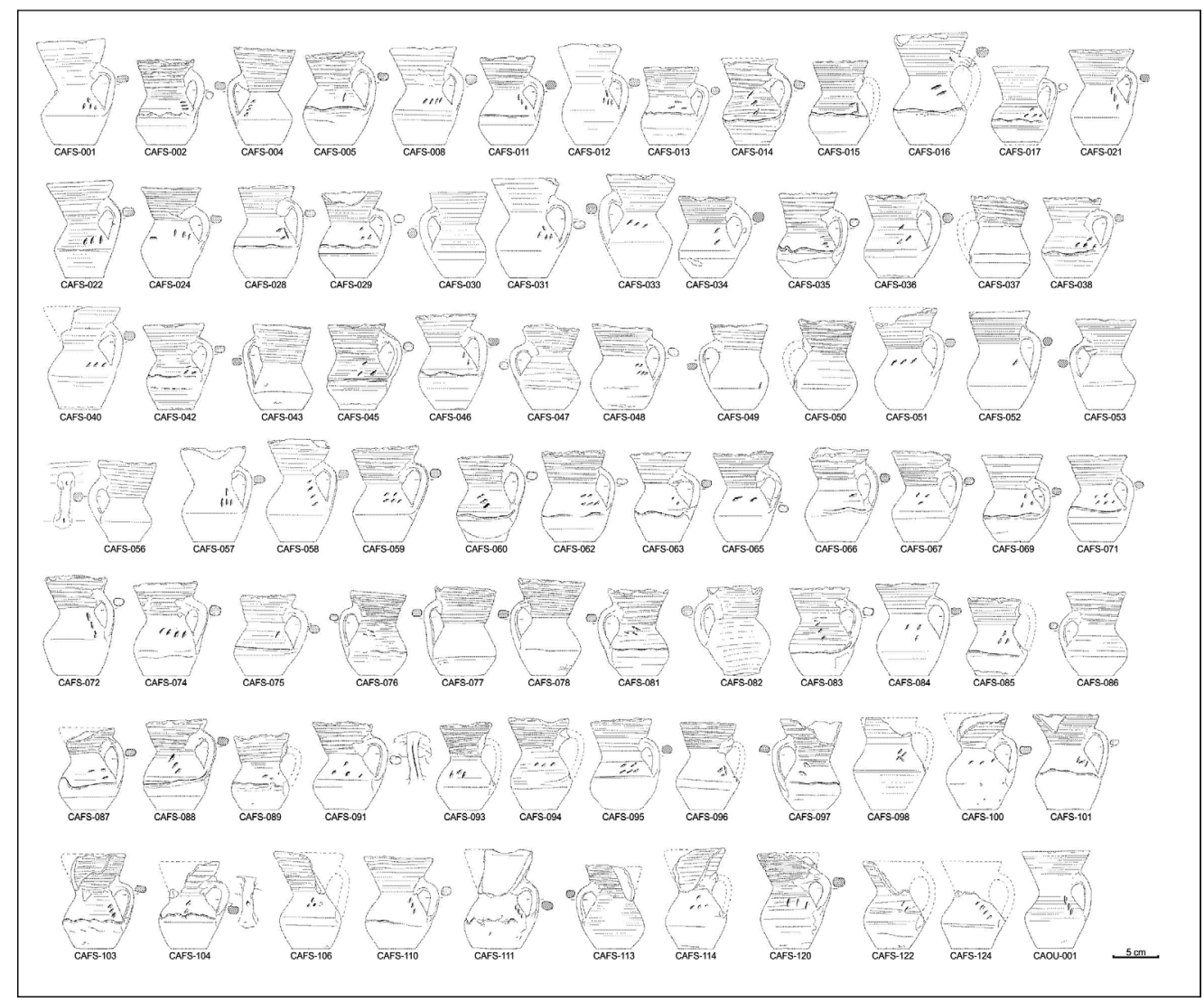

Fig. 4. Dibujo de las jarras de cuello troncocónico. 


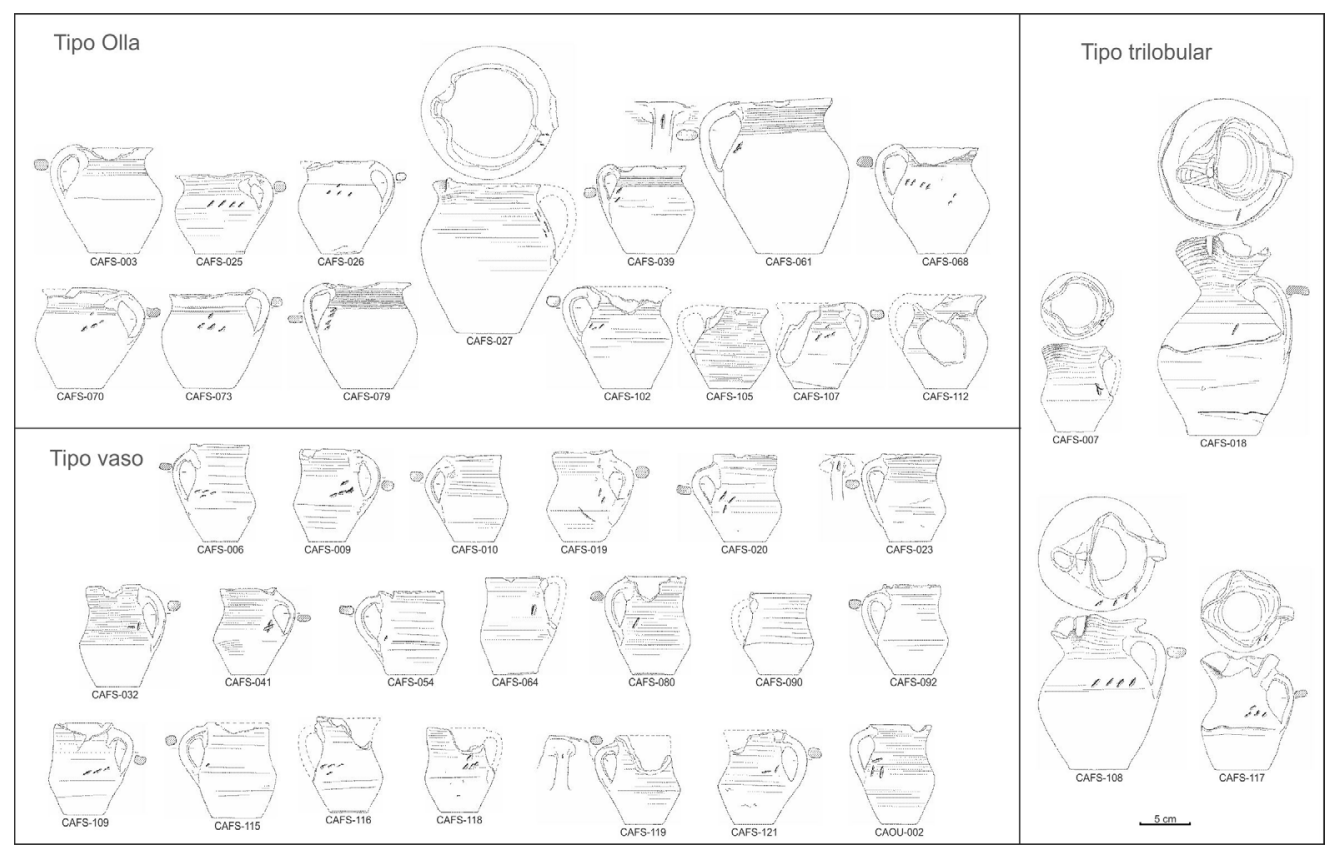

Fig. 5. Dibujo de las jarras tipo olla, vaso y boca lobulada.

Las asas presentan ciertas peculiaridades que merece la pena sintetizar. La forma se adapta al tipo de perfil, destacando 3 variantes en la forma del perfil: vertical (29 jarras), semicircular (28 jarras) o con hombro superior (42 jarras). Esta es la tendencia por tipos también. Un segundo aspecto curioso a tratar en relación con esta parte del recipiente es la inclinación de las asas, que aunque es predominante la vertical en número (38 jarras) y tipo, le sigue la inclinación lateral izquierda con más o menos inclinación (38 jarras) y un poco menor el número de recipientes con una inclinación lateral derecha (24 jarras). Este tipo de información nos puede ayudar a conocer el sentido del torno en el momento de colocar el asa o quizás si el alfarero era diestro o zurdo, siendo una información de interés para la posible identificación del número de alfareros o talleres que realizaron las jarras. 


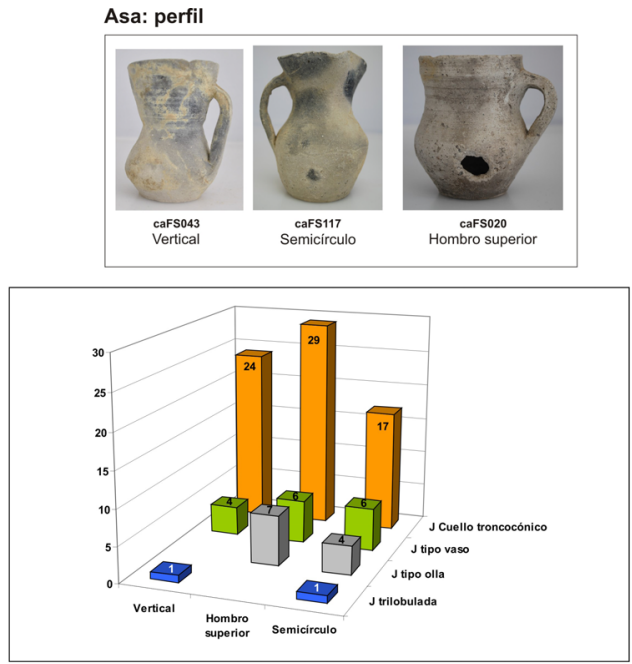

Inclinación del asa
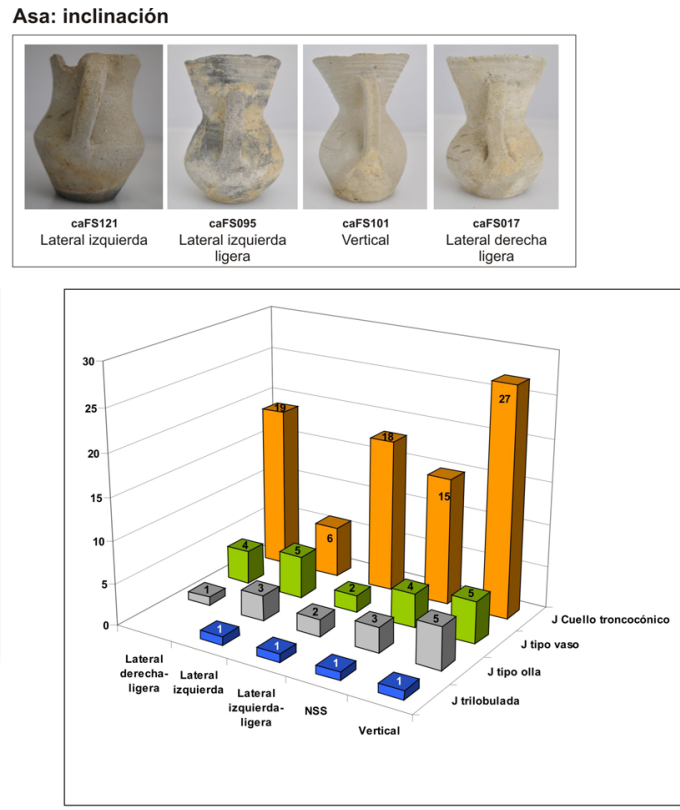

Perfil del asa

Fig. 6. Selección representativa de asas en las que se muestra su inclinación y morfología.

\section{Tratamiento de las pastas}

En lo que se refiere al tratamiento de las pastas, se detecta una enorme homogeneidad en el conjunto cerámico pues el 94\% de los recipientes posee pastas de coloración gris (gris blanquecino, gris claro perla, gris medio, gris oscuro) y sólo el 7\% presenta una cierta variedad de tonos en marrón, negro y rojo. El gris perla es el color predominante (84 jarras) y el tipo de desgrasante encontrado en todas las jarras es principalmente micáceo con algún cuarzo. A la hora de hacer grupos de pastas el color probablemente sea el criterio visual más acertado, pues la mayor parte de las jarras son de unas texturas compactas finas con desgrasantes finos distribuidos regularmente en la matriz arcillosa, y sólo en un número excepcional de jarras grises (blanquecinas y claro perla) pueden poseer texturas harinosas y jabonosas, y se puede observar algún desgrasante que puede alcanzar $2 \mathrm{~mm}$ muy puntualmente, en algún caso localizado en el asa (única parte hecha a mano) y no en el cuerpo de los recipientes. Así que la homogeneidad en el tratamiento de las pastas nos indica una manufactura quizás realizada por el mismo alfarero o taller, observando variaciones ligeras en el color, dependientes probablemente del horneado. Únicamente las jarras que no son grises 
parecen haber sido tratadas por una mano diferente. Si ponemos en relación el color con los morfotipos, se observan variaciones de tonos que podrían estar en relación con las diferencias cuantitativas entre grupos, pues entre J1 encontramos todos los colores, mientras que en el $\mathrm{J} 4$ sólo con 4 recipientes encontramos recipientes con pastas gris perla claro. Por lo cual se puede afirmar que no existen diferencias, al menos que podamos considerar significativas, entre tipos y grupos de pastas.

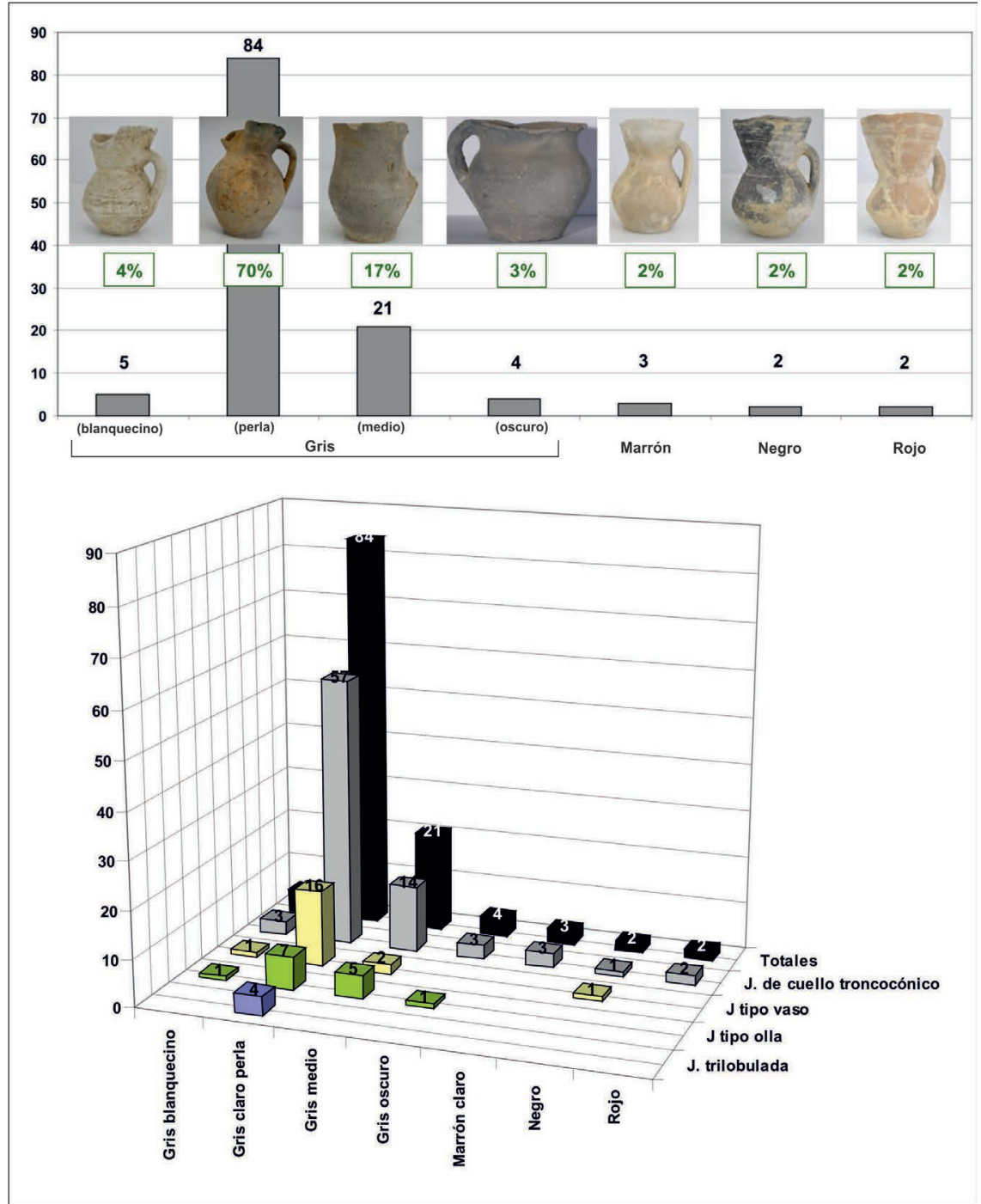

Fig. 7. Gráfica y esquema con los tipos de pastas documentados. 


\section{Huellas y grado de conservación de las jarras}

Las vasijas presentan huellas que pueden asociarse a diferentes estados de su biografía de fabricación, uso o deposición.

En primer lugar podemos tratar la más evidente, de carácter postdeposicional, pues la mayor parte de las jarras poseen una pátina blanquecina consecuencia de haber estado enterradas en una zona encharcada constantemente. Esta pátina es variable, tanto en superficie exterior como la interior y presenta en algunos casos la forma en la que debió estar posicionada la vasija hasta que la desenterraron. Tanto la pátina de las jarras como el hecho de que se conserven casi completas nos evidencia que las vasijas fueron depositadas cuidadosamente, estuvieron en un ambiente poco variable y no se movieron hasta su recuperación en 1960, a pesar de la datación de la tierra recuperada del interior de la vasija 73 del s. XVII ${ }^{27}$. De hecho las fracturas de algunas de ellas son relativamente frescas evidenciando una rotura posiblemente asociada a su recuperación reciente.

En segundo lugar, las huellas relacionadas con procesos de fabricación son las más fáciles de apreciar, por ejemplo, la presencia de manchas de color negruzco en la mayor parte de las jarras localizadas de forma puntual tanto en el interior como en el exterior son consecuencia de procesos irregulares de salida de oxígeno durante la cocción en el horno y de la posición que tuviese la vasija en su interior. Además, se han podido caracterizar algunos aspectos relacionados con la fase de modelado de las jarras, gracias a disponer de un número tan elevado de vasijas completas hemos podido establecer el grado de perfección del perfil en función de la simetría de los recipientes, tanto en su perfil como en su altura. El perfil de 70 vasijas es simétrico y de 30 sólo ligeramente asimétrico, y esta simetría u horizontalidad, en cambio, se documenta en altura en 36 vasijas aunque sólo 13 poseen una altura asimétrica u oblicua teniendo el resto una boca ligeramente oblicua, por lo que se podría concluir que el grado de habilidad de los alfareros que las manufacturaron es elevado. De forma puntual se aprecian pequeños detalles de manufactura, como alguna rebaba, huellas de unión de cuerpo y asa o panza-fondo, etc. Finalmente, no se aprecian restos asociables a un uso previo a la deposición de las jarras, por lo cual podríamos pensar que fueron fabricadas quizás para ser depositadas tal y como se encontraron. En otros yacimientos como en Franco 31, en Santiago de Compostela, o en Bordel, Padrón, se han encontrado restos

${ }^{27}$ Además de las muestras datadas en el edificio y el entorno, también se extrajo una pequeña muestra de sedimento del interior de la jarra número 73 con la intención de datar el momento de deposición de las jarras. Los resultados radiocarbónicos ofrecieron una cronología amplia pero mucho más tardía de lo esperado para las jarras, oscilando

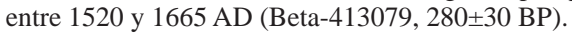


de hollín externo que permiten plantear un uso de las jarras como recipientes para calentar líquidos, siendo su función por lo tanto doble, por un lado cocina y por el otro, servicio de mesa ${ }^{28}$. Este tipo de restos no fueron encontrados en las jarritas de Augas Santas.

\section{Tratamiento de la decoración}

Finalmente, en lo que se refiere a las decoraciones, el primer rasgo llamativo es la elevada proporción de jarras decoradas en el conjunto, un total de 111 (91\%) y no se observan diferencias entre los morfotipos. Esto es un aspecto poco usual en los conjuntos de cerámica medieval publicados en la región, donde suele encontrarse decorado aproximadamente el $50 \%$ del material cerámico ${ }^{29}$, si bien es cierto, estos estudios siempre se realizan sobre material deficientemente conservado, es decir fragmentado y no sobre recipientes completos como el caso de Augas Santas Otro rasgo que convierte al conjunto de Augas Santas en único es la riqueza decorativa de sus jarras y la originalidad en uso de recursos muy sencillos para conseguirla.

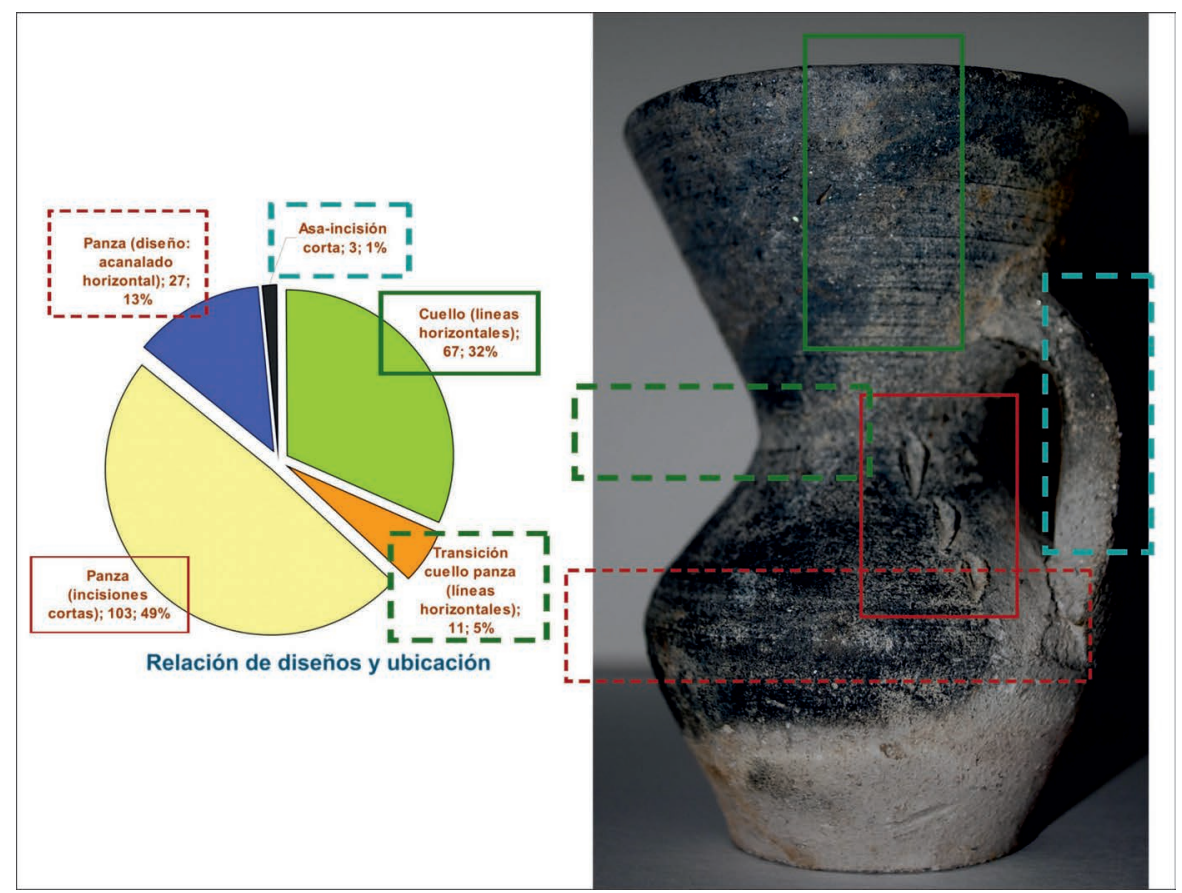

Fig. 8. Patrón decorativo documentado en las jarras de Augas Santas.

\footnotetext{
${ }^{28}$ ALONSO TOUCIDO, F. et al. (2013). “Cerámica en silos...”, op. cit.

29 Ibid.
} 
Se define un patrón espacial de la decoración (figs. 8 y 11) en el que los alfareros juegan con varios niveles formales para conseguir dicha riqueza, desde la localización de la decoración y la incorporación de recursos técnicos que habitualmente no son decorativos a través de los que se consigue un efecto decorativo, hasta el uso típico de las propias técnicas decorativas (figs. 9-10).

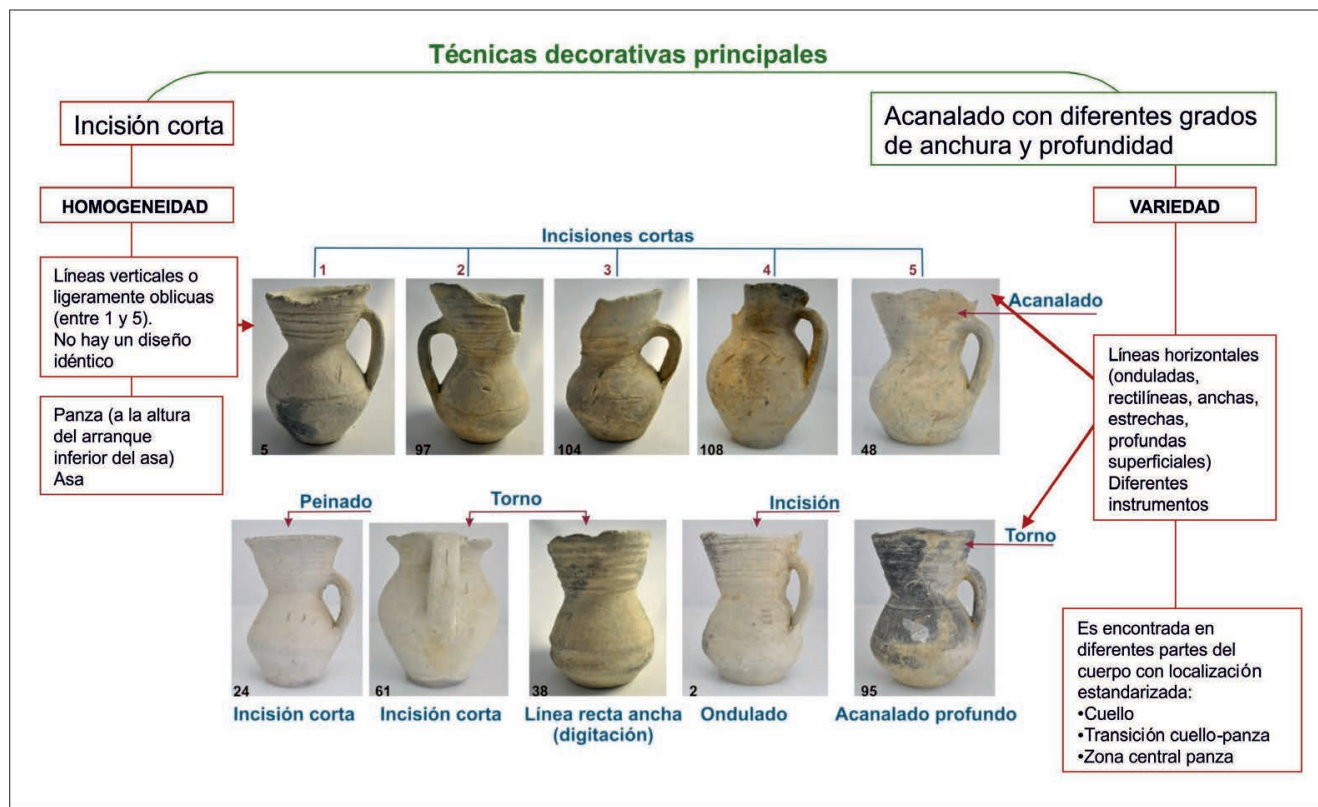

Fig. 9. Técnicas decorativas destacadas en las jarras de Augas Santas.

La forma en la que se puede definir la decoración de las jarritas es la siguiente:

1) Recurren a técnicas no decorativas para potenciar la decoración como por ejemplo el uso de huellas de torno localizadas en el cuello de las jarras (en el 55\% de las jarras).

2) Asimismo, algunos perfiles de recipientes son retocados en sus panzas, que son aplanadas en la zona de mayor expansión eliminando un efecto de carena por una forma en paréntesis (sólo en 3 jarras). Buscan en todo momento donde colocar cada uno de los efectos que se desean conseguir con la decoración, asignando cada tipo de motivo a una parte particular del cuerpo de la jarra.

3) El uso de elementos decorativos geométricos sencillos como líneas horizontales largas (rectilíneas o curvilíneas), líneas oblicuas o verticales cortas es frecuente en las jarritas. Las líneas acanaladas son diseños frecuentemente usados en la 
cerámica medieval gallega, si bien las incisiones cortas son relativamente abundantes, sobre todo aplicadas como decoraciones en las asas.

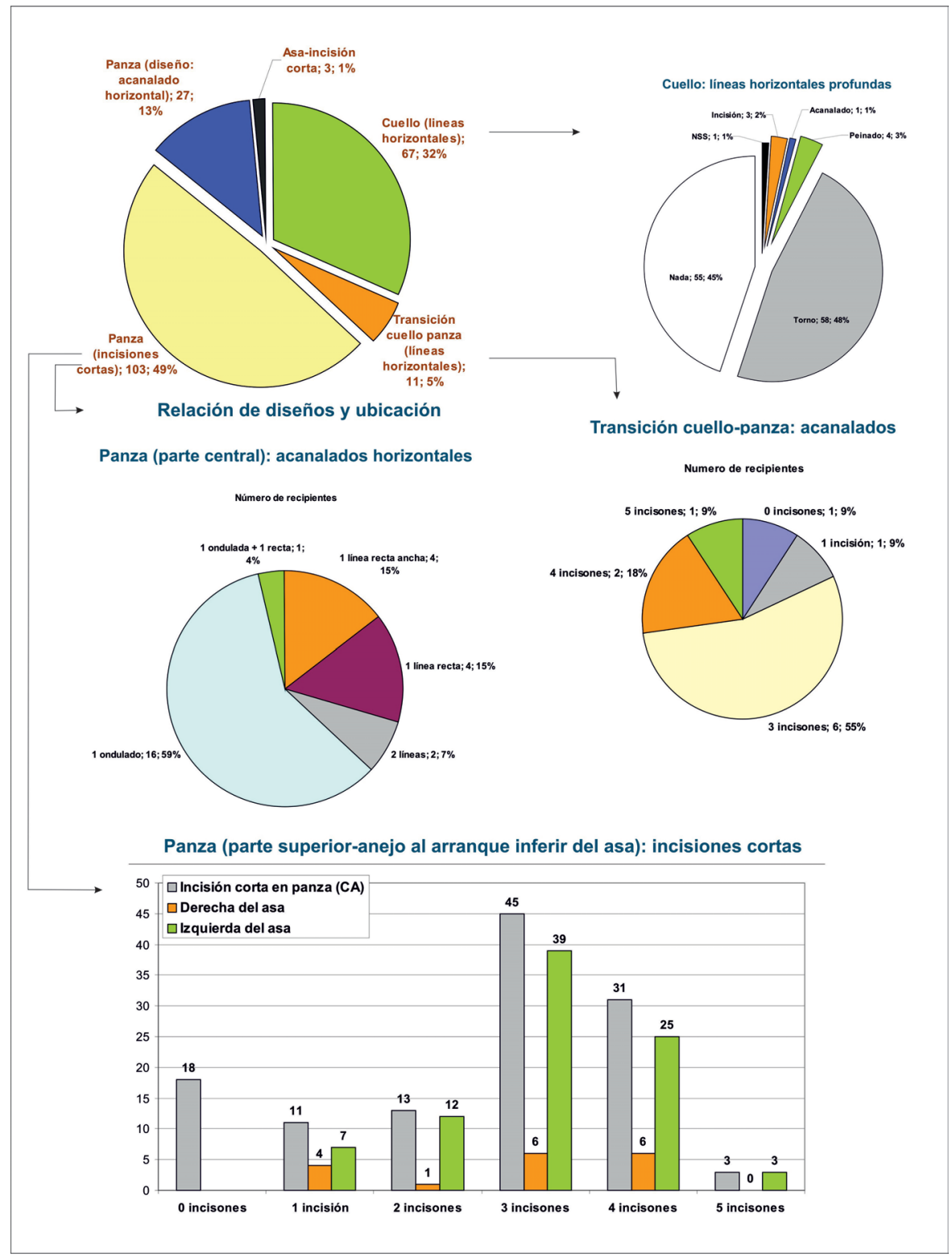

Fig. 10. Relación cuantitativa de técnicas decorativas en función de su localización en las jarras de Augas Santas. 
Francisco Alonso Toucido, Mª Pilar Prieto Martínez \& Anxo Rodríguez De Paz



Fig. 11. Esquemas decorativos de las jarritas de Augas Santas. 
Esta aplicación de técnicas decorativas típicas en la Edad Media en Augas Santas es utilizada de forma original en el conjunto de jarras:

- el acanalado con diferentes grados de anchura y profundidad utilizado en la transición cuello-panza (11 jarras) y la zona central de la panza (27 jarras poseen líneas onduladas o rectas);

- y la incisión corta (103 jarras), con marcas diferentes en la mayor parte de los vasos (entre 1 y 5 incisiones colocadas en la parte izquierda y en menor frecuencia derecha del arranque inferior de las asas, excepcionalmente se decoran las asas).

Estas marcas, las incisiones cortas, podrían ser un código aunque dado que no se dispone de información concreta sobre ellas, podríamos plantear varias posibles interpretaciones. Quizás pudieran estar haciendo referencia al alfarero o taller del que proceden las jarras. Una segunda hipótesis interpretativa es que cada huella haga alusión al propietario de la jarra que hace el encargo al alfarero, quizás con el afán de diferenciarla de las restantes jarras que se fabriquen en ese taller, mediante esta hipótesis se explicaría la escasa repetición de esquemas de incisiones y en los pocos casos en los que se da, se achacaría a jarras de propiedad familiar. Podríamos decantarnos quizás por esta segunda dado que, aunque son escasos los trabajos, en otras regiones, por ejemplo en las Islas Británicas, se han identificado marcas de propietario ${ }^{30}$, en el caso de la primera que planteamos, podría hacerse raro que hubiera una cantidad tan grande de talleres funcionando a la vez en la región y realizando jarras tan parecidas (quizás debería tener ello un impacto documental no disponible en la actualidad en ese caso). Una tercera hipótesis, complementaria de las anteriores, es que las marcas respondan a un sistema codificado que en su conjunto tenga un sentido, pero que nosotros no podemos descifrar todavía.

\section{CONCLUSIONES}

Nos encontramos con un depósito de cerámica de 128 jarras originalmente, colocado en un contexto complejo a nivel cronológico y funcional, en el que destaca una arquitectura de larga tradición en su uso (con constantes reformas relacionadas con los cambios religiosos y culturales de la zona).

30 GILCHRIST, R. (2012): Medieval life. Archaeology and the life course. The Boydell Press. Woodbridge Suffolk. pág. 226. 
Se trata probablemente de una producción en serie, que podría responder a un encargo posiblemente realizado por un mismo taller, y como consecuencia se observa desde el punto de vista formal una homogeneidad del conjunto con 4 grupos bien definidos en cuanto a morfología, asimismo sus pastas muestran unas características semejantes. Únicamente la decoración nos podría remitir a una 'cierta’ individualización de las jarras, quizás debido a que sus marcas puedan responder a la identificación de propiedad.

El conjunto de características morfotécnicas y decorativas del depósito, junto a la enorme cantidad de jarras y su excelente conservación relacionada con una colocación cuidada de las piezas nos permiten pensar que el depósito ha sido planificado como tal e incluso podría haber sido realizado por un mismo taller. Al que se le realizara un encargo comunitario con un objetivo ritual y sagrado. La propuesta cronológica para las jarras, correspondería con los momentos de última fase medieval constructiva en el edificio, es decir siglos XIII y XIV, tal vez se trata de un depósito fundacional, con motivo del inicio de la construcción de la basílica.

Este tipo de depósitos votivos todavía son poco conocidos en Europa, quizás dispongamos de uno en Galicia, en Lugo relacionado con un túmulo ${ }^{31}$, y son conocidos en las Islas Británicas algunos ejemplos también, sobre todo con restos faunísticos ${ }^{32}$, pero es un tema por investigar en profundidad. Sin duda alguna, por el momento Augas Santas es un caso especial que bien merece ser conocido en detalle.

\section{RECONOCIMIENTOS}

A Don Eduardo Fernández Rodríguez, párroco de Augas Santas (representante del Obispado de Ourense), quien nos proporcionó la casi totalidad de las jarras, 120 en total, y al Museo de Ourense, donde pudimos estudiar 2 de ellas.

El estudio de las jarras se enmarca en los proyectos siguientes:

Axudas do Programa de Consolidación e Estruturación de Unidades de Investigación Competitivas. Modalidade Grupos de Referencia Competitiva n. ${ }^{\circ}$ expte GRC2014/009 (GI-1919. SÍNCRISIS) Universidade de Santiago de Compostela. I.P.: M. García Quintela.

Tecnología y producción de la cerámica medieval de Galicia (MC-PTG). HAR2015-64441-P (Plan Nacional: Ministerio de Economía y Competitividad,

31 PRIETO MARTÍNEZ, M. P. et al. (2010). “La cerámica de dos túmulos...”, op. cit.

32 GILCHRIST, R. (2012): Medieval life. Archaeology..., op.cit. 
Convocatorias 2015, Proyectos EXCELENCIA y Proyectos RETOS, Dirección General de Investigación Científica y Técnica, Subdirección General de Proyectos de Investigación). I.P.: Pilar Prieto.

\section{BIBLIOGRAFÍA}

ALONSO TOUCIDO, F.; PRIETO MARTÍNEZ M. P.; RODRÍGUEZ RESINO A. (2013). "Cerámica en silos. Contextos medievales e modernos na rúa do Franco n 31. Santiago de Compostela”. Gallaecia, 32: 215-248.

Archivo Parroquial De Santa Mariña. 1962. Nota de limpieza de la fuente, p. 95.

BELLO DIÉGUEZ, J.M‥, SANJURJO SÁNCHEZ, J. y FERNÁNDEZ OSQUERA, D. (2008). "Los niveles medievales de la Torre de Hércules: caracterización arqueológica y datación mediante TL y OSL”. Férvedes, 5: 453-464.

BLANCO ROTEA, R.; GARCÍA RODRÍGUEZ, S.; MATO-FRESÁN, C.; SANJURJOSÁNCHEZ, J. (2015). "La Basílica da Ascensión y Os Fornos (Allariz, Ourense) y la cristianización de la arquitectura en la Antigüedad Tardía”. Estudos de Cuaternario, 12: 111-132.

BLANCO ROTEA, R., PRIETO MARTÍNEZ, M. P., BALLESTEROS ARIAS, P. y LÓPEZ GONZÁLEZ, L. F. (2010). “El despoblado de A Pousada: la formación de una aldea rural en la Alta Edad Media”. En Prieto Martínez, P. y Criado Boado, F. (Coord.), Reconstruyendo la historia de la comarca del Ulla-Deza (Galicia, España). Escenarios arqueológicos del pasado. TAPA 41. Santiago de Compostela: 111-120.

BONILLA RODRÍGUEZ, A. y CÉSAR VILA, M. (2005): “Excavación arqueológica en área en el solar de la antigua capilla y lazareto medieval de S. Lázaro (Santiago de Compostela, A Coruña)”. Gallaecia, 24: 219-242.

CÉSAR VILA, M. y BONILLA RODRÍGUEZ, A. (2011): “Síntesis de los materiales cerámicos procedentes del yacimiento de As Encrobas (Cerceda - A Coruña)”. En González, S. (ed.), La cerámica en Galicia: de los castros a Sargadelos. Actas del XIV congreso anual asociación de ceramología. Asociación de ceramología: 143-152.

CÉSAR VILA, M., BONILLA RODRÍGEZ, A. y LÓPEZ PÉREZ, Ma. C. (2010): “Aportaciones al conocimiento de la cerámica producida en la última fase de la Edad Media en Galicia”. En Crespo, M. y Martínez, R. (eds.), Metodología de análisis aplicada los estudios de cerámica tardoantigua y medieval de la Península Ibérica, Lobo Sapiens, León: 160-145.

CHAMOSO LAMAS, M. (1955): “Santa Marina de Augas Santas”. Cuadernos de Estudios Gallegos, X-20: 41-88.

FARIÑA BUSTO, F. (2000): “Xarriña Cerámica” Peza do Mes. Museo de Ourense, Setembro. FARIÑA BUSTO, F. (2002): Santa Mariña de Augas Santas, Grupo Marcelo Macías. Ourense. 
GILCHRIST, R. (2012): Medieval life. Archaeology and the life course. The Boydell Press. Woodbridge Suffolk.

MAPO (Museo Arqueolóxico de Ourense) (2008): Ourense. A Cidade, da orixe ao século XVI. Catálogo, Xunta de Galicia. Gráficas Garabal. A Coruña.

MARTÍNEZ CASAL, R. (2006): “A cerámica medieval da fortaleza da A Rocha Forte contribución ao seu estudo”, Gallaecia, 25: 187-225.

MARTÍNEZ PEÑÍN, R. (2013): “Los estudios de cerámica medieval en el noroeste de la Península: Galicia y Norte de Portugal”. Interconexôes. Revista de Ciencias Sociais, 1(1): 33-60.

MONTEAGUDO GARCÍA, L. (1967): “España Visigoda”. Noticiario Turístico, Suplemento 208: 3-63.

NIETO MUÑIZ, E.B. (2014): “Achádegos cerámicos na necrópole rupestres de San Vitor de Barxacova (Parada de Sil, Ourense)”. En De Man, A. e Tente, C (coords.) Estudos de cerâmica medieval. O norte e centro de Portugal, séculos IX a XII. Instituto de Estudos Medievais: 239-245.

PEREIRA MARIÓN, C. (1991): “As cerámicas medievais de Santa María de Augas Santas (Allariz, Ourense)”. Arqueoloxía, Informes 2, Campaña 1988, Xunta de Galicia: 347-350.

PRIETO MARTÍNEZ, M. P.; LANTES SUÁREZ, O.; VÁZQUEZ-LIZ, P.; MARTÍNEZCORTIZAS, A. (2010): "La cerámica de dos túmulos de Roza das Aveas (Outeiro de Rei, Lugo): Un estudio diacrónico del estilo y la composición”. BSAA, LXXVI: 27-62.

RODRÍGUEZ-CAO, C. (2009). "Escavación arqueolóxica en área nos soares n. 18-20 da rúa Colón e n 29 da rúa Cervantes da cidade de Ourense”. En Ourense, A Cidade, da orixe ao século XVI, Museo Arqueolóxico Provincial de Ourense, pp. 117-132.

SUÁREZ OTERO, J., GIMENO GARCÍA-LOMAS, R. y FARIÑA BUSTO, F. (1989): "La cerámica medieval en Galicia". En Gutiérrez González, J. A. y Bohigas, R. (eds.), La cerámica medieval en el norte y noroeste de la Península Ibérica. Aproximación a su estudio. Secretariado de Publicaciones, Universidad de León, León, pp. 285-301. 